\title{
Managing Human Resources Involved in Solving Atypical Situations: A Motivational Model
}

\author{
Grigore BELOSTECINIC \\ The Academy of Economic Studies of Moldova \\ Chisinau, Republic of Modova \\ belostecinic@yahoo.com
}

\author{
Lilia ȘARGU \\ University of European Studies of Moldova \\ Chisinau, Republic of Modova \\ lsargu@mail.ru
}

\begin{abstract}
Research actuality resumes from the fundamental role of the human factor in solving management issues, including contingency plans for unforeseen situations. The current management practices show just how human behavior can be unpredictable in settling the issue on time. The efficiency of the team member solving atypical situations is directly related to loyalty; level of competence; and the suitability of his, training psychological and physical working conditions and psychological climate in collective. Concepts reflect, in fact, the key factors that affect labor efficiency, such as desire; solving ability tasks and psychological conditions for this activity. In this context, the problem of the manager (team leader) is complex and she solved by attracting many different employees. However, the specificity and specificity of the human resources involved in the team consists in human behavior, the situation of inevitability, uncertainty and, as a consequence, in the modeling of processes that occur in systems with human elements. Nevertheless, despite a large number of studies on the study of human behavior, such processing methods are practically absent. In this context our research will propose formalization methods in searching for optimal management decisions (or acceptable) in this area. Considering above-mentioned elements, the purpose of this research formulated: mathematic description of the dependence based on effectiveness of personnel activity on the level of motivational measures of influence.
\end{abstract}

Keywords: solving atypical situations, motivation, the effectiveness of the employees' activity, team leader

\section{INTRODUCTION}

It is worth mentioning, that the process of motivation of the personnel science knows a significant number of works, where the respective problem, approached to from the psychological [1], sociological, managerial, economic, etc. point of view. Motivation meant to carry out a process of individual encouragement or other team members who will work to achieve the objectives for solving [2] the seemingly unforeseen situation as well as personal interests. Motivation is to closely human needs, because, as the notorious Soviet psychologists remarked A.N. Leontyev and S.L. Rubinstein, "the reason is an objective necessity".

Realization of individual needs of team members will be achieved through self-training and experience through unplanned situations and as for the team leader will be achieved through various mechanisms such as providing positive feedback with the result; promotion and career advancement; creating comfortable and safe working conditions etc. The mechanisms listed, implemented in accordance with psychophysical peculiarities of the subjects, are capable of producing significant impact on the formation of a high positive level of motivation and satisfaction engagement, forming thus a higher level of loyalty system. However, dependence on the effectiveness of the motivation to have a nonlinear character. The authors, studying the researches of Cozhokaru V.,[3] Burlaku N., Cindrea I.,[4] Popescu D.I.,[5] Birca A.,[6] Kotler Ph.,[7] Herse P., Kenneth H.B., Dewey E.J.,[8] Newcomb T.M., Turner R.H, Converse P.E.,[9] Stoner J. and Freeman R.E.,[10] Noel M.,[11] Druker P.,[12] Warren B. and Burt N.,[13], dealing with human resources, began to more closely examine these issues, which were the result of several publications.

\section{METHODS}

In order to research the author has identified as appropriate following methods: synthesis of theoretical components, the argument of the issues addressed, and generalization and tabulation hypothesis graphical representation theory proposed to substantiate the theory formula for success in solving atypical situations.

\section{RESULTS}

Finding ways to motivate team of experts in addressing and removing atypical situations. In the organizational context and characteristics to identify the arguments based on the 
To formalize obtained data further, numerical values

human factor as a factor stimulating for team leader solving atypical situations.

\section{DISCUSSION}

As the intensity, increases motivation, quality of the work changed along a bell-shaped curve initially advance, and then, after passing through the successful culmination gradually decreases. So, when work carried out in the most successful, mention this motivation optimal. Pattern identified by the first name of the Yerkes-Dodson law, has been experimentally confirmed and recognized repeatedly as one of the few indisputable objective psychological phenomena [14]. According to the second law of Yerkes-Dodson, the more difficult issue of performing the task, the low level of motivation is more to him stop.

The validity of these laws confirmed by studying the effectiveness of measures motivational team handling the atypical situations [15] arising in the process of achieving long-term strategy [16]. For employees involved in the provision of educational, research discovery has become an optimal level of incentive payments that amounts to $35-40 \%$ of salary. Meanwhile, staff with certain skills in research, this level was $50-60 \%$ of salary. The complexity of the tasks assigned to the employee took account of the basic salary.

The task for mathematical description of dependence on the effectiveness of the employees towards their motivation was determined when introducing new pay system for those involved in team solving atypical situations. So, it was analyzed staff work within a team with various tasks. The main tasks of each employee on the team classified according to their importance in order to achieve overall success. In addition, every month, every person drawn up a list of additional works proposed by the team leader or at the initiative of the individual. The experiment lasted one year. During the first received by members of that team constantly varied from $10 \%$ to $60 \%$ of salary. Each size of the first was valid for two months, after which it announced, that it increase by $10 \%$. It should also note that the team size personal payments for each member was strictly confidential. Disclosure or unauthorized attempt to obtain such information considered serious violation of the established rules and punished severely. Every month, the manager-leader of the team, according to the methods specifically developed was determined the level of satisfaction, commitment and motivation through verbal evaluations, consisting of a set of values, generally formed of 9 elements that within the scope of negative QL- and positive $\mathbf{Q L}+$ values:

$$
Q L=\{Q L-; H ; Q L+\}=\{\text { high negative }(I-)
$$

Higher negative average level (SM); Negative Medium (M); Lower negative average level (IM-); Neutral (low) (N); Lower positive average level (IM+); Positive Medium $(\mathrm{M}+)$; Higher level positive environment

$$
(S M+) ; \text { High positive }(I+)\}
$$

where assigned in accordance with the scale Harrington:

$$
\begin{aligned}
& N-\mathbf{0} ; \mathbf{I M} \pm- \pm \mathbf{0 . 2 9} ; \mathbf{M} \pm- \pm \mathbf{0 . 5 1} \\
& \mathrm{MS} \pm- \pm \mathbf{0 . 7 2} ; \mathbf{I} \pm- \pm \mathbf{1}
\end{aligned}
$$

And for evaluating effectiveness of the proposed team member $\mathbf{k}$ using the following formula:

$$
\begin{aligned}
& E_{\mathrm{k}}=D_{\text {eff }}\left[E_{k}^{B A S I C}+R_{k}^{S U P L}\right] \\
& E_{\mathrm{k}}^{\text {BASIC }}=\bar{W} \frac{1}{\bar{N}_{k}} \sum_{i=1}^{N_{k}}\left(\bar{s}_{i}^{k} \bar{q}_{i}^{k}\right) \\
& E_{k}^{S U P L}=\widetilde{W} \frac{1}{\widetilde{N}_{k}^{k}} \sum_{i=1}^{N_{k}}\left(\begin{array}{ll}
\Sigma_{i}^{k} & q_{i}^{k}
\end{array}\right)
\end{aligned}
$$

Where,

$E_{\mathrm{k}^{-}}{ }^{-}$the effectiveness of the overall index $\mathbf{k}$ team member; $E_{\mathrm{r}}$

$D_{\text {eff }}$ - defuzzification procedure of fuzzy values, obtained as a result of the calculation;

$E_{k}^{\text {BASIC }}$ - effectiveness of "basic" activities of $\mathbf{k}$ team member;

$E_{k}^{S U P L}$ - effectiveness of the "supplementary" activities of $\mathbf{k}$ team member;

$\bar{N}_{k}$ - number of basic functions of $\mathbf{k}$ team member in accordance with the job description;

- meaning (critical) type and the basic functions of $\mathbf{k}$ team member; $\bar{s}_{i}^{k}$

$\bar{q}_{i}^{k}$ - quality of performance team member $\mathbf{k}$ in $\mathbf{i}-$ basic types of tasks;

$N$ - the total number of additional works carried out in the department;

$\tilde{N}_{k}$ - the number of additional works performed k employees;

$\tilde{z}_{i}^{k}$ - complexity of $\mathbf{j}$ additional work performed by $\mathbf{k}$ employees; $\tilde{z}_{i}^{k}$

$\widetilde{q}_{i}^{k}$ - quality of performance $\mathrm{k}$ ja additional works team member;

$\bar{W}$ and - the weight coefficients reflecting the "contribution" The basic functions and respectively additional work performed by $\mathbf{k}$ team member to assess the effectiveness of its activities: $\tilde{W}$ coefficients $\bar{z}_{\tilde{E}}^{k}$ and $\tilde{z}_{i}^{k}$ were determined 
using the modified method of classifying non-strict [14] the main tasks of the team members, respectively, of additional work carried out in team period.

$$
\begin{aligned}
& \bar{W}=\left(\sum_{k} \bar{N}_{k}\right) /\left(\sum_{k}\left(\bar{N}_{k}+\bar{N}_{k}\right)\right) \\
& \tilde{W}=\left(\sum_{k} \tilde{N}_{k}\right) /\left(\sum_{k}\left(\bar{N}_{k}+\bar{N}_{k}\right)\right)
\end{aligned}
$$

Quality of completing tasks $\bar{q}_{i}^{k}$ and $\tilde{q}_{i}^{k}$ and positive estimated values the set of $Q L^{+}(1)$ :

$$
\begin{aligned}
& Q L^{+}=\{\text {Reduced }(N) ; \text { Lower level }(I M) ; \\
& \text { medium }(M) ; \text { Higher level }(S M) ; \text { tall }(I)\}
\end{aligned}
$$

Values in (7) estimated in accordance with the position of affiliation:

$$
\begin{aligned}
& \{N(0 ; 0 ; 0.15 ; 0.25) ; \\
& I M(0.15 ; 0.25 ; 0.35 ; 0.45) ; \\
& F(0.35 ; 0.45 ; 0.55 ; 0.65) ; \\
& S M(0.55 ; 0.65 ; 0.75 ; 0.85) ; \\
& I(0.75,0.85,1,1)\}
\end{aligned}
$$

Where $\boldsymbol{X} \boldsymbol{X}\left(a_{1}, a_{2}, a_{3}, a_{4}\right)$ - Trapezoidal fuzzy numbers in which $a_{1}$ and $a_{4}$ are abscissas of lower base; $a_{2}$ and $a_{3}$ abscissas of upper base of the trapezoid.

For fuzzy value calculations special software was applied [17] Method "center of gravity" has been applied for the defuzzification of calculated fuzzy value [18] In order to interpret the values obtained was used as the assumption that the motivational influence on the result of two factors impact acting in the opposite direction. On the one hand, measures increase the loyalty motivating team members, and on the other hand, it is the most powerful psychological factor that distract the employee from its functional responsibilities. The person begins to think more not out how to do those jobs better, but how to get more bonuses based on motivational incentive program.

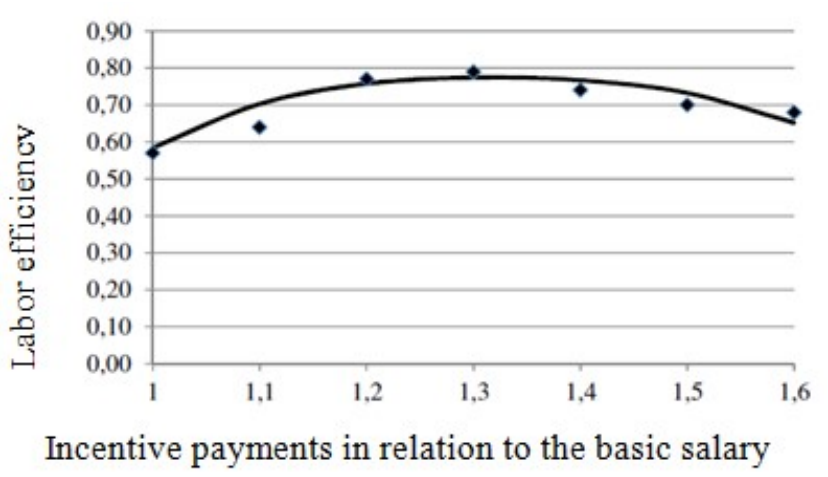

a)

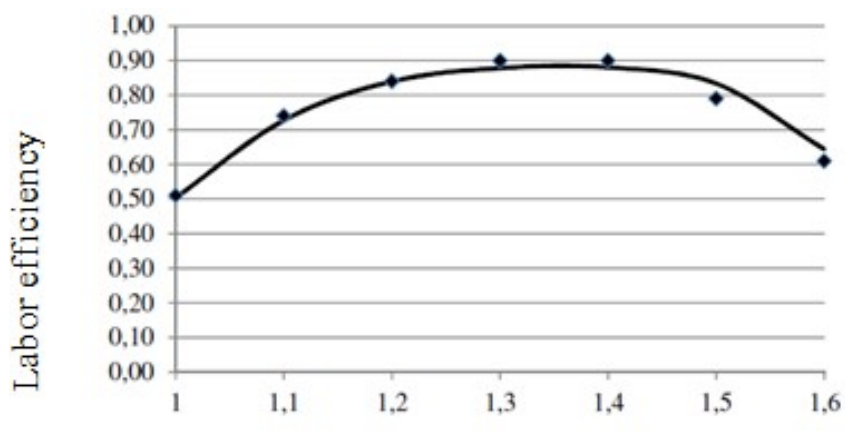

Incentive payments in relation to the basic salary

b)

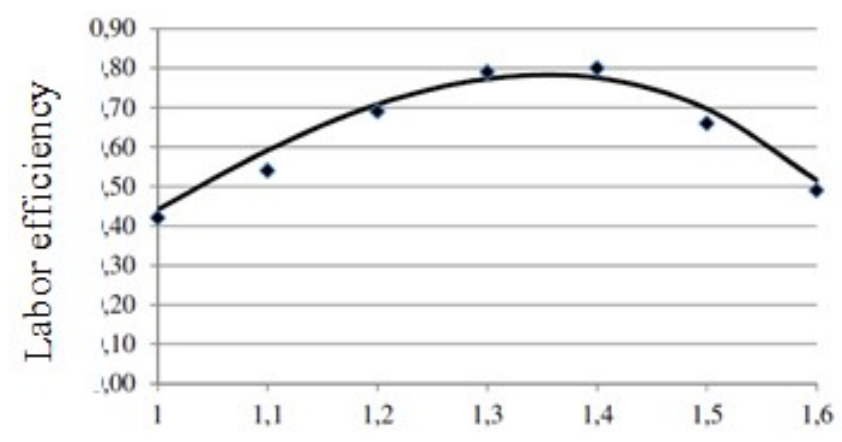

Incentive payments in relation to the basic salary

c)

Fig. 1. Dependence typical labor of the efficiency team member

In this context, it was proposed that: the dependencies of staff loyalty $f_{L}$ and psychological preparation $f_{R}$ (focus on successful completion of the work) level of motivation to be approximated with functions type $\mathrm{S}$ :

$$
\begin{gathered}
f_{\mathbf{L}}\left(M ; a_{L} ; b_{L} ; \bar{L}\right)=\bar{L} /\left(\bar{L}+e_{L}^{-a(M-b)}\right) \\
f_{R}\left(M ; a_{r} ; b_{r} ; \bar{R}\right)=\bar{R}-\bar{R}\left(\bar{R}+e_{r}^{-a(M-b)}\right)
\end{gathered}
$$




$$
E_{M}=f_{L} * f_{R}
$$

\section{Where}

$f_{\mathrm{L}}\left(\mathrm{M} ; \mathrm{a}_{\mathrm{L}} ; \mathrm{b}_{\mathrm{L}} ; \dot{\mathrm{K}}_{11}\right)$ is the function of loyalty;

$f_{\mathrm{L}}\left(\mathrm{M} ; \mathrm{a}_{\mathrm{r}} ; \mathrm{b}_{\mathrm{r}} ; \dot{\mathrm{K}}_{26}\right)$ - function of psychological training (orientation towards the successful implementation of the activity);

\section{$E M$ - function motivational effectiveness of measures;}

$M$ - the intensity of motivation (motivational influence measures);

$L \%$ - the maximum level of employee loyalty motivational values;

$P \%$ - the psychological preparation of the team members in the absence of motivational values;

$a_{L}$ and $a_{R}$ - numerical parameters of functions loyalty and psychological training;

$L B$ - coefficient values corresponding to a level such that loyalty motivational team member reaches half of its maximum value; value,

$b_{R}$ - coefficient corresponding to the motivation level

Experimental data obtained by the manager - leader were approximated by type functions. Typical results of approximation to work with different levels of complexity are shown in Fig. 1.

Approximation to work with different levels of complexity are shown in the Fig. 1.

Dependence typical labor efficiency team member where the main tasks are the following categories:

$$
\begin{aligned}
& a-\text { high }: \bar{L}=1 ; a_{L}=10 ; b_{L}=0,9 ; \overline{\mathrm{R}}=0,8 ; a_{R}=9 ; \\
& b_{R}=1,77 ; k_{c o p}=0,97 ; \\
& b-\text { medium }: \bar{L}=1 ; a_{L}=12 ; b_{L}=0,98 ; \overline{\mathrm{R}}=0,9 ; \\
& a_{R}=16 ; b_{R}=1,66 ; k_{c o p}=0,98 ; \\
& c-l o w: \bar{L}=0,9 ; a_{L}=7 ; b_{L}=0,99 ; \overline{\mathrm{R}}=0,9 ; \\
& a_{R}=10 ; b_{R}=1,64 ; k_{c o p}=0,96
\end{aligned}
$$

High values of correlation coefficient obtained between the theoretical and experimental values ( $k_{\text {cor }} \epsilon[0,92 ; 0,98]$ ) facilitates the conclusion regarding the correctness of the hypothesis.

Theoretical dependencies created allowed the identification of values "optimal motivation" for different employees. The values obtained can be use today as standards for incentive payments. The average increase in labor efficiency constituted, in this case, $36 \%$ (Table 1 ).

TABLE I. INCREASE LABOR EFFICIENCY TEAM MEMBER, \% OF THE AMOUNT CORRESPONDING TO THE ABSENCE OF PAYMENTS MOTIVATING

\begin{tabular}{|l|l|l|l|}
\hline \multicolumn{2}{|c|}{ indicator } & \multicolumn{3}{|c|}{$\begin{array}{c}\text { complexity } \\
\text { basic responsibilities, \% }\end{array}$} \\
\cline { 2 - 4 } & \multicolumn{1}{|c|}{ high } & \multicolumn{1}{|c|}{ average } & \multicolumn{1}{c|}{ low } \\
\hline $\begin{array}{l}\text { Average value of increase labor } \\
\text { efficiency }\end{array}$ & 29 & 38 & 42 \\
\hline $\begin{array}{l}\text { Average value of incentive } \\
\text { payments, when } \\
\text { maximum efficiency of labor is } \\
\text { achieved }\end{array}$ & 27 & 32 & 44 \\
\hline
\end{tabular}

It should note that the nature of experimental dependencies obtained mainly corresponds laws Yerkes - Dodson: employee work in the efficiency diagram contains the optimum level reached as quickly as difficult team member is responsible (Table 1).

The behavior of team members, however, was nonstandard. Graphs efficacy created for them did not have a pronounced maximum level compared with other members' chart. In addition, the value of the highest efficacy achieved in an atypical complexity of work performed. From making a more detailed study, it was found that the average income per family member (VMMF) team members was 3-6 times higher than in others, so increasing motivation for motivational level rise is less pronounced than other employees (Fig. 2).

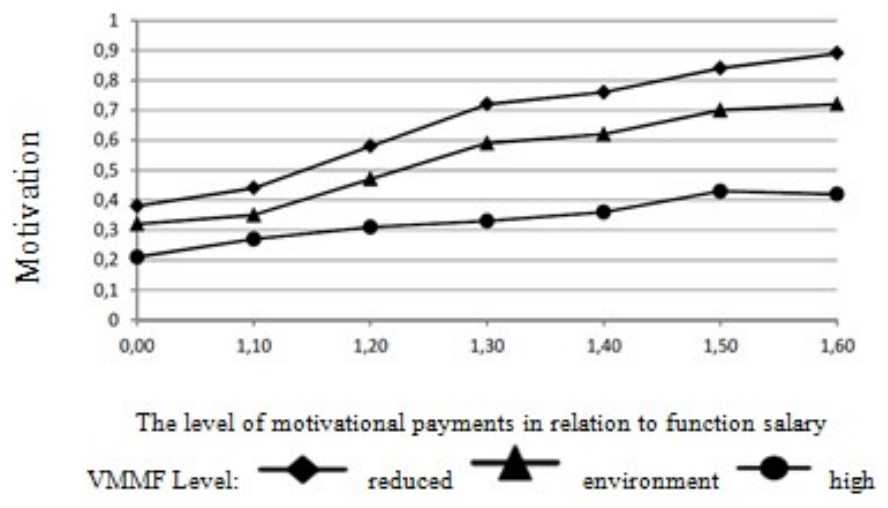

Fig. 2. The diagrams on the team's motivation different levels of average income per family member

According to data we found that the motivation is not directly proportional to the amount of incentive payments and the nature of this dependence differs for various categories of experts who VMMF different. This fact should considered in the search for the optimal value of incentive payments: to stimulate high-level experts with average income for a family member, it is necessary to apply other methods of motivational influence (mainly intangible). It should also be noted that the formulas (1) - (10) not only allows numerical evaluation of the effectiveness of measures motivational adopted human resource management, but also allows you to select the ways to further improve work with the anthropogenic subsystem. 
For example, if the maximum effectiveness of the "basic” value $E_{k}^{\text {basic }} a \mathbf{k}$ team member is systematically lower than $\bar{W}$ in the case of quite high satisfaction, commitment and motivation, then, most likely, this team member is not suitable in terms of competence and / or psychophysical characteristics, to perform the tasks assigned. It is necessary that possibilities (capabilities) of team members and tasks be performed compliance.

If the maximum effectiveness of the "basic" activity is systematically, lower than $\bar{W}$ for most employee's division, then, most likely it due to lack of conditions for effective work. Working within the team, this attested to those experts who have certain skills in the use of Information Technologies and was unable to perform their duties in good time and in full.

Additional work that repeated systematically may also show poor distribution of functional responsibilities. Such activities must be included in the list of mandatory tasks and be met by a particular employee.

\section{CONCLUSIONS}

The human factor considered a key solution in any situation regardless of the extent of the problem of character or her circumstances. Providing leading process management in special situations becomes a growing problem given current strategic long-term uncertainty [19] To ensure efficiency of the process, the author identified methods of teamwork from expert professionals, concrete issues organized by the selection list manager leading professional experts [20] on certain dimensions. Professional team training is one of the conditions that will contribute to solving atypical situations, and the second component determined by the competence leader.

Therefore, effective management of human resources is the most important factor determining the success of any business expert teams. One of the main methods of management motivational impact on team members, together forming the anthropogenic subsystem, but also institutional and information management.

The approach proposed in this study to describe the motivation of human resources management enables qualitative assessment of the effectiveness of efforts also making recommendations for further improving the effectiveness of social work component of teamwork in solving atypical situations.

Experimental verification of the model settlement cases of atypical situations through expert team proves the correctness of the proposed approach. Implementation of the recommendations obtained by using mathematical model of motivational management of human resources will contribute to increasing the effectiveness of the respective teams for atypical situations.

The human factor is the main element in management activity. Organizational management processes [21] in the case of arising atypical issues done by human resources involved solving the problem. Troubleshooting depends on the professionalism and competence of those involved in problem solving.

\section{REFERENCES}

[1] S. S. Stepanov, "Popular psychological encyclopedia", M: Eksmo, 2005, $672 \mathrm{p}$.

[2] L. Shargu, "The solution of the atypical situations through the human factor", Modern Paradigms in the Development of the National and World Economy USM, Chisinau 02-03 November 2018, pp. 41-48.

[3] N. Burlacu and V. Cozhokaru, "Management", ASEM, Chisinau, 2000, $350 \mathrm{p}$.

[4] I. Cindrea, "Human Resources Management", Sibiu, 2008 [Electronic resource]. Available at: tp://didu.ulbsibiu.ro/myself $1 / x-$ resurse/resurse/r_1570925323926_Managementul_resureselor_umane/1/ Managementul_resurselor_umane.\%20Cindrea_I.pdf.

[5] D.I. opescu, "General management of the company", ed. 4th., ucharest, ASE, 2017, $250 \mathrm{p}$.

[6] A. Bîrcă, "Human Resources Management Manual", ASEM Publishing House, Chisinau, 2005.

[7] Ph. Kotler, "Kotler Strategic Management: Best Practices and Methods", 3rd ed., M: Alpina Publisher, 2016, 132 p.

[8] P. Hersey, H. B. Kenneth, E. J. Dewey, "Management of organizational behavior: leading human resources", 2001.

[9] T. M. Newcomb, R. H. Turner, and P.E.Converse, "Social Psychology: the Study of Human Interaction", New York: Holt, Rinehart and Winston, 1965.

[10] J. Stoner and R.Freeman, "Management", New Jersey, Ed. Prentice Hall, Englewood Cliffs, 1992, 66 p.

[11] M. Noel, "The leader or the art of driving", Bucharest, Teora Publishing House, 2000, $122 \mathrm{p}$.

[12] P. Druker, "About the profession of manager", Meteor Press Publishing House, Bucharest, 1998.

[13] B. Warren and N. Burt, "Leaders. Business Tech International Press Publishing House", Bucharest, 2000, 102 p.

[14] A. N. Leontyev, "Activity. Consciousness. Personality", M.: Meaning, $2005,352 \mathrm{p}$.

[15] L. Shargu, "Management functions in atypical situations", Knowledge Horizons - Economics, Bucharest, Romania, September, 2019, vol. 11, no. 3, pp. 52-60 [Electronic resource]. Available at: http://orizonturi.ucdc.ro/arhiva/KHE\%20nr.\%203\%20-\%202019/8.\% 20MANAGEMENT\%20FUNCTIONS\%20IN\%20ATYPICAL\%20SIT UATIONS.pdf.

[16] L. Sargu, "Management of atypical Situations through the quality management system", in the: Collective Monograph, the management of innovative economic development ENTITIES in 2 vol., Higher School of Social and Economic Prezevorsk, Polska, 2018.

[17] A. P. Rotshtein, "Influence of defuzification methods on the tuning speed of a fuzzy model", Cybernetics and system analysis of Shtovba, 2002, no. 1, pp. 28-35.

[18] B. Kosko, "Fuzzy systems as universal approximators", IEEE Transactions on Computers, 1994, vol. 43, no. 11, pp. 1329-1333.

[19] L. Shargu, "The strategic nature of the management of atypical situations", in a collective monograph, Financial management of current market research, Moscow: LLC Rusyns, 2018, 145 p., pp. 57-75.

[20] Iu. Valeeva and L. Sargu, "Methodological approaches to the management of client orientation in the development of management sistem of services", in the European Journal of Accounting, Finance and Business, 2019, vol. X, issue XX [Electronic resource]. Available at: http://www.accounting-management.ro/index.php?pag=show content\&issue $=20 \&$ year $=2019$.

[21] L. Shargu and C. Coman, "Method of Handling the Atypical Situations Management through the Informational System", in the Saudi Journal of Business and Management Studies, September, 2019, vol. 4, issue 9 [Electronic resource]. Available at: https://scholarsmepub.com/sjbms-49. DOI: $4 \mathrm{i} 09.003$ 